\title{
ARTICLE
}

Physiology and Biochemistry

\section{Placental mobilization of free fatty acids contributes to altered materno-fetal transfer in obesity}

\author{
Birgit Hirschmugl $\mathbb{1}^{1,2} \cdot$ Simone Perazzolo ${ }^{3,4} \cdot$ Bram G. Sengers ${ }^{3,4} \cdot$ Rohan M. Lewis $\mathbb{D}^{4,5} \cdot$ Michael Gruber $^{1} \cdot$ \\ Gernot Desoye ${ }^{1}{ }^{1} \cdot$ Christian Wadsack ${ }^{1,2}$
}

Received: 24 April 2020 / Revised: 8 January 2021 / Accepted: 1 February 2021 / Published online: 26 February 2021

(c) The Author(s) 2021. This article is published with open access

\begin{abstract}
Background Metabolic changes in obese pregnant women, such as changes of plasma lipids beyond physiological levels, may subsequently affect fetal development in utero. These metabolic derangements may remain in the offspring and continue throughout life. The placenta mediates bidirectional exchange of nutrients between mother and fetus. The impact of prepregnancy obesity on placental transfer of lipids is still unknown.

Objective We aimed to examine materno-to-fetal free fatty acid (FFA) transfer by a combined experimental and modeling approach. Flux of ${ }^{13} \mathrm{C}$-labeled FFA was evaluated by ex vivo perfusion of human placentae as a function of prepregnancy obesity. Mathematical modeling complemented ex vivo results by providing FFA kinetic parameters.

Results Obesity was strongly associated with elevated materno-to-fetal transfer of applied ${ }^{13} \mathrm{C}$-FFA. Clearance of polyunsaturated ${ }^{13} \mathrm{C}$-docosahexaenoic acid (DHA) was most prominently affected. The use of the mathematical model revealed a lower tissue storage capacity for DHA in obese compared with lean placentae.

Conclusion Besides direct materno-to-fetal FFA transfer, placental mobilization accounts for the fetal FA supply. Together, with metabolic changes in the mother and an elevated materno-fetal FFA transfer shown in obesity, these changes suggest that they may be transmitted to the fetus, with yet unknown consequences.
\end{abstract}

\section{Introduction}

Obesity has become a global burden as the prevalence has continuously increased in the last decades. Consequently, increasing numbers of women in the reproductive age are

Supplementary information The online version contains supplementary material available at https://doi.org/10.1038/s41366021-00781-x.

Christian Wadsack

christian.wadsack@medunigraz.at

1 Department of Obstetrics and Gynaecology, Medical University of Graz, Graz, Austria

2 BioTechMed-Graz, Graz, Austria

3 School of Engineering, Bioengineering Research Group, University of Southampton, Southampton, UK

4 Institute for Life Sciences Southampton, University of Southampton, Southampton, UK

5 University of Southampton, Faculty of Medicine, Southampton General Hospital, Southampton, UK also affected by this disease. Obesity is defined by an excess of body weight for height and is expressed by a body mass index (BMI) score of $>30 \mathrm{~kg} / \mathrm{m}^{2}$ [1]. Obesity and pregnancy are linked with a number of pregnancy complications for the mother. In particular, obese mothers are at higher risk of developing hypertension, preeclampsia, and gestational diabetes [2]. Maternal obesity also increases risk for a delayed labor onset and slow labor progress that often results in unplanned cesarean delivery [3]. Prepregnancy obesity can affect the growth of the fetus in utero. Notably, elevated percentage of neonatal body fat and concurrently normal birth weight were reported [4]. Infants of obese mothers are at a higher risk to be born large for gestational age $[5,6]$ and subsequently develop childhood obesity $[6,7]$, a predictor of adulthood obesity [8]. Thus, infants of obese women are at a high risk of entering into the generational spanning obesity-diabetes cycle.

Facing childhood obesity as a serious public health problem, it would be necessary to disclose the origin of fetal overgrowth and understand the mechanisms resulting in childhood obesity. 
In this context, some studies report alterations in maternal plasma lipid profile in obese women such as elevated triglyceride levels [9, 10], or increased insulin resistance [11-13] compared to lean pregnant women. However, the immediate contribution of maternally derived metabolic changes to excessive lipid accumulation in fetal adipose tissue is still elusive. All exchange processes between the maternal and fetal circulation are mediated by the placenta, and therefore appropriate placental function is crucial for fetal development. The fetus is dependent on the supply of maternal long-chain polyunsaturated fatty acids (LC-PUFA) that are specifically required for an appropriate development of the brain and neuronal system [14]. Recent studies have shown that metabolic changes, as a result of obesity even before pregnancy, are associated with elevated placental lipid accumulation [15-17] and impaired mitochondrial function that is further related to increased oxidative stress in placental tissue [16, 18]. Alterations in placental expression of fatty acid transport proteins (FATP), fatty acid translocase (CD36), or fatty acid binding proteins (FABP) were reported in relation to obese conditions in mice and humans [9, 19, 20]. FATP and CD36 are membrane proteins which are involved in the cellular uptake of free fatty acids (FFA). Once FFA are taken up by the placenta, they are bound to FABP and further processed to membrane phospholipids, used for ATP production in mitochondria, or esterified to triacylglycerols as the intermediate storage form in cells [21]. Although lack of direct evidences, maternal obesity alters at least placental lipid homeostasis which eventually may alter lipid supply to the fetus.

The driving mechanisms underlying the transplacental FFA transfer need to be elucidated. The question arises if and how the placenta is affected and regulated under pathophysiological metabolic conditions, such as maternal obesity. In this study with a sole focus on the placenta, we aimed to examine alterations in placental FFA transfer in respect to maternal prepregnancy obesity. Therefore, we used the ex vivo dual placental perfusion approach to study direct transfer of ${ }^{13} \mathrm{C}$-labeled FFA across placental tissue of obese and lean mothers. In addition, the mathematical model for placental FFA transfer mechanisms, previously published by Perazzolo et al. [22] was applied to elucidate further key factors underlying the obtained experimental materno-to-fetal FFA transfer results in placentas of obese compared to lean mothers.

\section{Materials and methods}

\section{Study subjects}

Women either with a prepregnancy body mass index (BMI) $<25 \mathrm{~kg} / \mathrm{m}^{2}$ (lean, $n=8$ ) or BMI $\geq 30 \mathrm{~kg} / \mathrm{m}^{2}$ (obese, $n=7$ ) were included in this study. Gestational age of all women was $>38$ weeks and all delivered by elective cesarean section. According to the medical records, participating women were fasted overnight and did not take any medication. All women were normotensive. Gestational diabetes was excluded by oral glucose tolerance test between gestational week 24 and 28. The study followed the declaration of Helsinki was approved by the ethics committee of the Medical University of Graz (EK No. 24-529 ex 11/12) and written informed consent was signed by all study participants.

\section{Reagents and equipment}

For all placental perfusion experiments Dulbecco's modified eagle medium (DMEM, phenol red free, Gibco, UK) was used. DMEM was mixed with Earl's buffer $(6.8 \mathrm{~g} / \mathrm{L}$ $\mathrm{NaCl}, 0.4 \mathrm{~g} / \mathrm{L} \mathrm{KCl}, 0.14 \mathrm{~g} / \mathrm{L} \mathrm{NaH}{ }_{2} \mathrm{PO}_{4}, 0.2 \mathrm{~g} / \mathrm{L} \mathrm{MgSO}_{4} \bullet 7$ $\mathrm{H}_{2} \mathrm{O}, 0.2 \mathrm{~g} / \mathrm{L} \mathrm{CaCl} 2,2.2 \mathrm{~g} / \mathrm{L} \mathrm{NaHCO}_{3}$, all Merck, Darmstadt, Germany) in a 3:1 medium buffer ratio containing amoxicillin (250 mg/L, Sigma-Aldrich, Steinheim, Germany), dextran FP40 (10 g/L, Serva, Heidelberg, Germany), $2 \mathrm{~g} / \mathrm{L}$ glucose (Merck, Darmstadt, Germany), and essential fatty acid free bovine serum albumin (BSA) (5 g/L, SigmaAldrich, Seinheim, Germany).

Perfusion medium containing ${ }^{13} \mathrm{C}$-labeled free fatty acid mix $\left({ }^{13} \mathrm{C}\right.$-FFA mix) was prepared under a stream of argon to prevent fatty acid oxidation. The albumin to FFA molar ratio was 0.77 , which is in the physiological range in the systemic circulation of a pregnant women [9, 23]. Preparation details and FFA final concentrations are shown in Supplementary Table 1.

\section{Ex vivo dual placental perfusion setup}

The ex vivo placental perfusion set up used in this study was adapted and technically extended based on the approach of Schneider et al. [24]. Briefly, within $30 \mathrm{~min}$ after delivery of the placenta a corresponding chorionic artery and vein pair supplying one intact cotyledon was cannulated. The cannulated cotyledon was immediately flushed with pre-warmed $\left(37^{\circ} \mathrm{C}\right)$ perfusion medium, thereafter this cotyledon and surrounding tissue was placed in the pre-warmed perfusion chamber. Finally, the cannula of the fetal artery was connected to the reservoir containing perfusion medium kept at $37^{\circ} \mathrm{C}$ in a water bath. Gas levels in the medium were adapted by a gas exchange device (Living Systems, St. Albans, VT, US) operated with 95\% $\mathrm{N}_{2}$ and $5 \% \mathrm{CO}_{2}$ during the experiment. The fetal circulation was always perfused with fresh medium (open circulation), which allowed us to distinctly follow each FA released from the placenta. A constant fetal artery inflow of $4 \mathrm{~mL} / \mathrm{min}$ was set up by using a magnetic pump (Codan, Salzburg, 
Austria). Volume loss was monitored within the first $30 \mathrm{~min}$, thereafter every hour at the fetal venous outflow port. Each cotyledon displayed at least $95 \%$ fetal flow recovery. The backflow pressure in the fetal vascular system was recorded by a micro catheter pressure sensor (Millar, US) inserted into the fetal arterial cannula. Experiments with vessel back pressure lower than 65 mbar were considered as successful [25].

Next, maternal circulation was set up by inserting three rounded needles into the intervillous space of the cotyledon. During the experiment, medium in maternal circulation was gassed with $5 \% \mathrm{CO}_{2}, 20 \% \mathrm{O}_{2}$, and $75 \% \mathrm{~N}_{2}$ by applying a gas exchange device. The maternal flow rate was kept at $8 \mathrm{~mL} / \mathrm{min}$. Within the first phase of perfusion experiment, antipyrine $(100 \mu \mathrm{g} / \mathrm{mL}$, Sigma-Aldrich, Schnelldorf, Germany) was added to the maternal reservoir and perfusion was operated by open circulations. Antipyrine crosses the placental barrier by passive diffusion and serves as quality control substance for the size of the materno-to-fetal exchange area [26].

Samples from maternal artery and fetal vein outflow (perfusates) at the time points $0,10,20$, and $30 \mathrm{~min}$ were collected, centrifuged at $3000 \mathrm{rpm}$ at $4{ }^{\circ} \mathrm{C}$ for $10 \mathrm{~min}$, and stored at $-80^{\circ} \mathrm{C}$ until antipyrine concentrations were determined.

Thereafter, the maternal reservoir was switched to perfusion medium containing ${ }^{13} \mathrm{C}$-FFA mix $(200 \mathrm{~mL}, 0.5 \%$ $\mathrm{BSA}$ ) and the system changed to closed circuit at the maternal side. Samples of both circuits were collected at 0 , 10, 20, 30, 60, and $90 \mathrm{~min}$, centrifuged and stored at $-80^{\circ} \mathrm{C}$. Finally, maternal reservoir was switched to media containing $0.5 \%$ BSA with an open circuit configuration for a further $30 \mathrm{~min}$. At the end of the experiment, wet weight of the perfused cotyledon was determined and is designated as perfused tissue mass $(\mathrm{ptm})$ in the calculations and statistics section.

In order to assess viability of the perfused cotyledon, maternal and fetal perfusates were sampled $(1.5 \mathrm{~mL}$, via sampling port) during the experiment every $30 \mathrm{~min}$. Oxygen $\left(\mathrm{pO}_{2}\right)$, carbon dioxide $\left(\mathrm{pCO}_{2}\right), \mathrm{pH}$, lactate production, and glucose consumption were measured by a blood gas analyzer (Radiometer, Copenhagen, Denmark). LabVIEW based recording software (Beko engineering, Graz, Austria) was used to register data sets obtained by the blood gas analyzer, the magnetic pumps, and the pressure sensor.

\section{Antipyrine quantification by HPLC}

Antipyrine concentrations of maternal and fetal perfusates were determined by HPLC as described by Annola et al. [27]. Briefly, $100 \mu \mathrm{L}$ of perfusate was mixed with $100 \mu \mathrm{L}$ methanol (Sigma-Aldrich, Schnelldorf, Germany), vortexed and centrifuged at $12000 \mathrm{rpm}$ for $15 \mathrm{~min}$. Upper phase
$(150 \mu \mathrm{L})$ was transferred to a new tube and mixed with $150 \mu \mathrm{L}$ acetonitrile (Sigma-Aldrich, Schnelldorf, Germany), again vortexed and centrifuged. A series of standards with antipyrine concentrations from $5 \mu \mathrm{mol} / \mathrm{L}$ to $1 \mathrm{mmol} / \mathrm{L}$ was prepared as described above. Antipyrine concentrations were determined by HPLC (Knauer, Berlin, Germany), which was equipped with an aquasil $150 \times 2.15 \mu$ column (Thermo scientific, Waltham, MA, USA) and an UV detector (Knauer, Berlin, Germany). Either $10 \mu \mathrm{L}$ sample or standard was injected, followed by an isocratic flow $(0.2 \mathrm{~mL} / \mathrm{min})$ run with $20 \mathrm{mmol} / \mathrm{L} \quad \mathrm{KH}_{2} \mathrm{PO}_{4}$ (Merck, Darmstadt, Germany) in water mixed 1:1 with acetonitrile. Antipyrine peaks were detected at $255 \mathrm{~nm}$ and antipyrine concentrations were calculated according to the obtained standard curve.

Antipyrine fetal/materno ratio (FM ratio) was calculated and only perfusion experiments with a FM ratio $\geq 0.3$ in $30 \mathrm{~min}$ were considered as a successful experiment and further processed.

\section{Fatty acid analysis by gas chromatography-mass spectrometry (GC-MS)}

Total lipids, out of $1 \mathrm{~mL}$ perfusion medium, were extracted in methanol/tert-butyl methyl ether/water (1.5/5/1.25) as described by Matyash et al. [28]. Samples were spiked with C15:0 FFA $(3.75 \mathrm{nmol} / \mathrm{mL})$ as internal standard. The organic phase was dried in a vacuum centrifuge. Lipids were dissolved in $500 \mu \mathrm{L} \mathrm{CHCl}_{3} /$ methanol (1/1). FFA were determined out of total lipid extracts, as previously published by Fuchs et al. [29] with some modifications. Briefly, solvent of lipid extracts $(200 \mu \mathrm{L})$ were evaporated, resolved in $50 \mu \mathrm{L}$ of a pentafluorobenzyl bromide solution $(3.4 \%$ in acetonitrile), and $10 \mu \mathrm{L}$ of $\mathrm{N}, \mathrm{N}$-diisopropyl ethanolamine. Samples were incubated at room temperature for $10 \mathrm{~min}$, evaporated under a gentle stream of nitrogen, finally resuspended in $50 \mu \mathrm{L}$ of hexane and used for FFA analysis.

All ${ }^{13} \mathrm{C}$-labeled FFA carried ${ }^{13} \mathrm{C}$ isotopes on each $\mathrm{C}$-atom position. The labeling efficiencies were $97 \%$ atoms for DHA and $99 \%$ atoms for the other FFA, as specified by the manufacturers. In order to verify retention time and mass of the individual ${ }^{13} \mathrm{C}$-FFA, we performed a trial measurement of each single ${ }^{13} \mathrm{C}$-FFA by GC-MS, equipped with a quadrupole triple-axis detector (Agilent Technologies, Santa Clara, CA, USA), run on negative ion chemical ionization and selected-ion monitoring (SIM) mode (a list of masses given in Supplementary Table 1). FFA species, including ${ }^{13} \mathrm{C}$-labeled FFA were detected by retention time (according to mixed FFA standards) and mass, after loss (m-1) in SIM scan mode. To quantify specific FFA, areas under the curve (AUC) were calculated by Mass Hunter (Agilent Technologies, Santa Clara, CA, USA). Ratios between internal standard $(3.75 \mathrm{nmol} / \mathrm{mL} \mathrm{C15:0} \mathrm{FFA)} \mathrm{and}$ 
each individual FFA species were calculated. Additionally, FFA in perfusates containing $0.5 \%$ BSA (blank, without ${ }^{13} \mathrm{C}$-FFA mix) were determined, and obtained values (mainly low levels of 16:0 and 18:0) were subtracted from measured FFA concentration of each sample. No corrections were made for natural abundance of ${ }^{13} \mathrm{C}$ since each $\mathrm{C}$ atom carried an ${ }^{13} \mathrm{C}$-isotope of used labeled FFA and a defined mass shift depending on FFA chain length of +16 (palmitic acid), +18 (oleic and linoleic acid), or +22 (DHA), respectively. We assumed equal response factors for all labeled and un-labeled FFA. All reagents were purchased form Sigma-Aldrich, Steinheim, Germany, if not other stated.

\section{Mathematical modeling}

The mathematical model used here has been described in detail previously [22]. Briefly, a compartmental modeling approach was adopted to predict the concentrations involved in the experimental setup: maternal artery, maternal vein, syncytiotrophoblast, and fetal vein. All compartments were assumed to be well-mixed, while transfer between compartments was described by facilitated diffusion. In this study, the model was used specifically to represent the ${ }^{13} \mathrm{C}$-DHA uptake and transfer in comparison with the experimental data. By fitting the model to the data, four parameters were estimated: the maximum uptake flux parameter for the microvillous membrane $\left(\mathrm{v}_{\mathrm{MVM}}\right)$, the maximum delivery flux parameter for the basal membrane $\left(\mathrm{v}_{\mathrm{BM}}\right)$, the syncytiotrophoblast metabolic accumulation rate constant $\left(\mathrm{k}_{\mathrm{acc}}\right)$ and the syncytiotrophoblast metabolic release rate constant $\left(\mathrm{k}_{\mathrm{rel}}\right)$. The parameters were determined separately for each of the lean and obese subjects, averaged for each group and tested for significant difference between groups. To test the predictive capabilities of the model, an additional independent experiment was performed. The first phase of the experiment was performed with ${ }^{13} \mathrm{C}$-FFA mix containing $0.3 \mu \mathrm{mol} / \mathrm{L}{ }^{13} \mathrm{C}$-DHA (see also Supplementary Table 1), after $120 \mathrm{~min}$, the experiment was continued by switching back to closed loop perfusion with a new reservoir concentration of $1.5 \mu \mathrm{mol} / \mathrm{L}{ }^{13} \mathrm{C}$-DHA in the FFA mix (five times the dose given at time zero). The parameters extracted from the first phase of the experiment $(0-120 \mathrm{~min})$ were then used for the prediction of the second phase from $120 \mathrm{~min}$ to $210 \mathrm{~min}$.

\section{Calculations and statistics}

Antipyrine transfer was calculated as followed.

$$
\text { FM ratio }=\frac{f v}{m a}
$$

fv: concentration in fetal vein; ma: concentration in maternal artery

Materno-to-fetal FFA transfer was normalized to mean perfused tissue mass ( $25 \mathrm{~g}$, Supplementary Table 2), and calculated as clearance.

$$
\text { Clearance }=\frac{\frac{f v}{p t m} * 25}{m a} * Q f
$$

fv: concentration in fetal vein; Qf: fetal flow rate $(\mathrm{mL} / \mathrm{min})$; ma: concentration in maternal artery; ptm: perfused tissue mass $(\mathrm{g})$

Isotopic enrichment (\%) was calculated as followed.

$$
\text { Isotopic enrichment }(\%)=\frac{f v}{(f v+f v p l)} * 100
$$

fv: ${ }^{13} \mathrm{C}$-FFA concentration in fetal vein; fv pl: placental released non-labeled FFA concentration in fetal vein

${ }^{13} \mathrm{C}$-FFA tissue uptake (\%) was calculated as followed.

$\operatorname{Uptake}(\%)=\frac{(m r t 0-m r t 90-f r t 90)}{m r t 0} * 100$

$\mathrm{mr}$ t0: ${ }^{13} \mathrm{C}$-FFA absolute quantity in the maternal reservoir at time point $0 \mathrm{~min}$; $\mathrm{mr}$ t90: ${ }^{13} \mathrm{C}$-FFA absolute quantity in the maternal reservoir at time point $90 \mathrm{~min}$; fr t90: ${ }^{13} \mathrm{C}$-FFA absolute quantity in the fetal reservoir at time point $90 \mathrm{~min}$

Data are presented as mean \pm SD. Statistical analysis was performed by two-way ANOVA with Bonferroni post hoc test $\left({ }^{13} \mathrm{C}\right.$-FFA clearance), non-parametric group comparison Mann-Whitney $U$ test (model parameter for ${ }^{13} \mathrm{C}-\mathrm{FFA}$ and study population characteristics) and Kruskal-Wallis with post hoc Dunn's test (FFA placental release in comparison to ${ }^{13} \mathrm{C}$-FFA transfer). $P$ values $<0.05$ were considered significant. For statistical analysis GraphPad Prism 7.0 (GraphPad Software, La Jolla, CA) and IBM SPSS Statistics (version 23) was used.

\section{Results}

\section{Evaluation of materno-to-fetal FFA transfer by combining experimental and mathematical model approaches}

The placental transfer for palmitic acid (PA, 16:0), oleic acid (OA, 18:1), linoleic acid (LA, 18:2n6), and docosahexaenoic acid (DHA, 22:6n3) was examined by placental ex vivo perfusion. Stable isotope ${ }^{13} \mathrm{C}$-labeled PA, OA, LA, and DHA together with a combination of nonlabeled FFA were applied to perfusion medium containing albumin. All ${ }^{13} \mathrm{C}$-labeled FFA were already detectable in fetal effluent after $10 \mathrm{~min}$ perfusion (Fig. 1A). The long chain polyunsaturated ${ }^{13} \mathrm{C}$-DHA clearance was significantly 

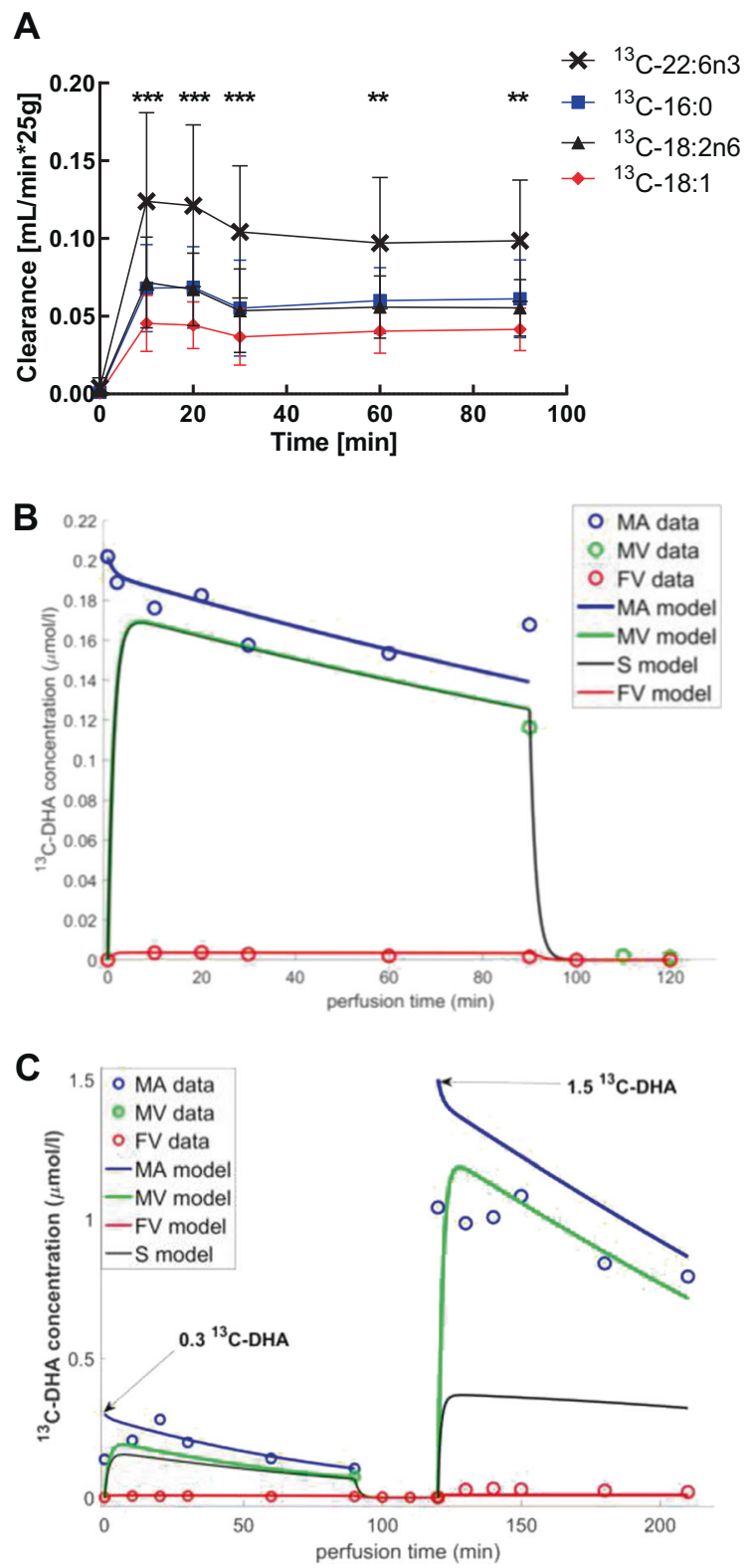

more pronounced at all measured time points than the clearance for ${ }^{13} \mathrm{C}-\mathrm{PA},{ }^{13} \mathrm{C}$-LA, and ${ }^{13} \mathrm{C}-\mathrm{OA}$.

Therefore, we implemented ${ }^{13} \mathrm{C}$-DHA transfer data in our previously published mathematical model [22]. The mathematical model was able to effectively match the current experimental data for ${ }^{13} \mathrm{C}$-DHA presented in one representative plot (Fig. 1B). A robust model must be able to work under a range of different conditions. In order to test the reliability of the mathematical model, the perfusion protocol was adapted and the experiment extended with a second higher dose of ${ }^{13} \mathrm{C}$-DHA $(1.5 \mu \mathrm{mol} / \mathrm{L}$; Fig. 1C). The correspondence between model and experiment demonstrated how the model was able to predict reasonably wellperfusion experiments for different ${ }^{13} \mathrm{C}$-DHA input conditions based on the kinetics estimated during the first phase
Fig. 1 Clearance of ${ }^{13} \mathrm{C}$-labeled FFA and model prediction fitting the perfusion data for the ${ }^{13} \mathbf{C}$-DHA placental transfer. (A) Clearance of ${ }^{13} \mathrm{C}$-labeled palmitic acid (16:0), oleic acid (18:1), linoleic acid (18:2n6), and docosahexaenoic acid (22:6n3) was examined by placental perfusion $(n=13)$. Kinetic of FFA transfer was investigated utilizing a closed (recirculated) maternal and open (nonrecirculating) fetal circulation. Mean $\left( \pm\right.$ SD) ${ }^{13} \mathrm{C}$-FFA clearance was calculated at time points $0,10,20,30,60$, and $90 \mathrm{~min}$. Two-way ANOVA and Bonferroni post hoc test were performed, $* * p<0.01$ and $* * * p<0.001$ indicate significant differences between ${ }^{13} \mathrm{C}$-DHA and other ${ }^{13} \mathrm{C}$-FFA. (B) Circles represent the experimental data, solid lines represent the model predictions for the ${ }^{13} \mathrm{C}$-DHA concentration of one lean subject. Blue represents the maternal artery (MA); green represents the maternal vein (MV); black represents the syncytiotrophoblast compartment $(\mathrm{S})$; red represents the fetal vein $(\mathrm{FV})$. Note that maternal vein concentrations were measured only at $t=90 \mathrm{~min}$, whilst no experimental data were available for the tissue concentrations. Model parameter from one representative experiment is illustrated. (C) Model prediction when $0.3 \mu \mathrm{moL} / \mathrm{L}{ }^{13} \mathrm{C}$-DHA was added at time zero, followed by $1.5 \mu \mathrm{moL} / \mathrm{L}$ at $\mathrm{t}=120 \mathrm{~min}$. The total perfusion time was 210 min. Circles represent the experimental data, solid lines for the model predictions. Blue represents the maternal artery (MA); green represents the maternal vein ( $\mathrm{MV}, \mathrm{t}=90 \mathrm{~min}$ only); black represents the syncytiotrophoblast compartment ( $\mathrm{S}$, no experimental data available); red represents the fetal vein (FV). The model parameters were estimated solely based on the data from the first phase of the perfusion experiment $(0-120 \mathrm{~min})$ and then applied to predict the second phase of the experiment (120-210 min). Note that the initial concentration values used for the model were based on the initial dose administered. This particular experiment was performed once.

of the experiment. Note that due to the experimental variation in this particular case the initial concentrations used in the model were based on the initial dose administered, which is higher than the first concentrations measured in the experiment. The kinetic model parameters estimated for ${ }^{13} \mathrm{C}$-FFA in the lean and obese group are presented in Table 1. For each examined ${ }^{13} \mathrm{C}$-FFA (PA, OA, LA, and DHA), the $\mathrm{v}_{\mathrm{MVM}}$ was significantly $(p<0.01)$ higher than the $\mathrm{v}_{\mathrm{BM}}$ by over two orders of magnitude, implying that the effective permeability of the MVM was much greater than from the BM. Comparing the lean control and obese group there was a trend for a difference in the accumulation into metabolism parameter $\mathrm{k}_{\mathrm{acc}}$ for ${ }^{13} \mathrm{C}$-DHA, but it was not significant $(p=$ $0.08)$. The estimated parameter $k_{\text {rel }}$ for metabolic release of ${ }^{13} \mathrm{C}$-FFA during the experiment was zero in both cases meaning no relevant release back in the system after sequestration by the metabolic pool in the $90 \mathrm{~min}$ experimental period.

Reliability of obtained ex vivo perfusion experiments is shown by monitoring specific metabolic parameters in accordance with recommended practice [25] (Supplementary Table 2).

\section{Effect of maternal prepregnancy obesity on trans-placental FFA transfer}

Study population $(n=15)$ was classified according to maternal prepregnancy BMI into a lean (BMI $18.5-24.9 \mathrm{~kg} /$ 
Table 1 Estimated model parameters for ${ }^{13} \mathrm{C}$-FFA of lean and obese experiments.

\begin{tabular}{|c|c|c|c|c|c|c|}
\hline & $\mathrm{v}_{\text {MVM }}$ & $\mu \mathrm{mol} / \mathrm{min})$ & $\mathrm{v}_{\mathrm{BM}}(\mu \mathrm{m}$ & $1 / \min )$ & $\mathrm{k}_{\mathrm{aac}}($ & $/ \min )$ \\
\hline${ }^{13} \mathrm{C}-16$ & & & & & & \\
\hline Lean & 17.1 & $( \pm 14.9)$ & 0.008 & $( \pm 0.006)$ & 0.65 & $( \pm 0.58)$ \\
\hline $\begin{array}{l}\text { Obese } \\
{ }^{13} \mathrm{C}-18\end{array}$ & 18.5 & $( \pm 26.2)$ & 0.014 & $( \pm 0.008)$ & 1.58 & $( \pm 2.28)$ \\
\hline Lean & 25.5 & $( \pm 18.5)$ & 0.132 & $( \pm 0.340)$ & 0.54 & $( \pm 0.77)$ \\
\hline Obese & 27.9 & $( \pm 51.8)$ & 0.012 & $( \pm 0.006)$ & 0.73 & $( \pm 1.26)$ \\
\hline${ }^{13} \mathrm{C}-18:$ & $2 n 6$ & & & & & \\
\hline Lean & 16.0 & $( \pm 23.3)$ & 0.044 & $( \pm 0.107)$ & 0.59 & $( \pm 1.05)$ \\
\hline $\begin{array}{l}\text { Obese } \\
{ }^{13} \mathrm{C}-22:\end{array}$ & $\begin{array}{c}11.9 \\
6 \mathrm{n} 3\end{array}$ & $( \pm 19.0)$ & 0.005 & $( \pm 0.004)$ & 0.34 & $( \pm 0.39)$ \\
\hline Lean & 0.083 & $( \pm 0.059)$ & 0.00004 & $( \pm 0.00003)$ & 0.86 & $( \pm 0.96)$ \\
\hline Obese & 0.124 & $( \pm 0.113)$ & 0.00004 & $( \pm 0.00002)$ & 0.34 & $( \pm 0.17)$ \\
\hline
\end{tabular}

$\mathrm{v}_{\mathrm{MVM}}$ represents the maximum uptake flux parameter for the MVM ( $\mu \mathrm{mol} / \mathrm{min}) ; \mathrm{v}_{\mathrm{BM}}$ is the maximum flux parameter for the BM ( $\mu \mathrm{mol} /$ $\min ) ; \mathrm{k}_{\mathrm{acc}}$ depicts the metabolic accumulation rate constant $(1 / \mathrm{min})$. The rate constant $\mathrm{k}_{\mathrm{rel}}$ for metabolic release of labeled fatty acid from the placental tissue is equal to zero and not reported in the table. Mean $( \pm \mathrm{SD})$ of lean control $(n=8)$ and obese $(n=7)$ groups are shown. There are no statistically significant differences between control and obese groups but there was a trend for a difference in ${ }^{13} \mathrm{C}-22: 6 \mathrm{n} 3 \mathrm{k}_{\mathrm{acc}}$ $(p=0.08)$. Non-parametric group comparison (Mann-Whitney $U$ test) was performed.

$\mathrm{m}^{2}, n=8$ ) and obese group (BMI $\geq 30 \mathrm{~kg} / \mathrm{m}^{2}, n=7$ ). The obese group was matched for maternal age and gestational age. Neither placental weight nor birth weight or ponderal index was significantly different between lean and obese groups (Supplementary Table 3).

The impact of maternal prepregnancy obesity on FFA transfer to the fetus in late pregnancy was investigated by comparing placental clearance of ${ }^{13} \mathrm{C}-\mathrm{PA},{ }^{13} \mathrm{C}-\mathrm{OA},{ }^{13} \mathrm{C}-\mathrm{LA}$, and ${ }^{13} \mathrm{C}$-DHA of lean $(n=7)$ and obese $(n=6)$ women (one experiment in each group was excluded from statistical analysis due to missing FFA measurement at one single time point). Independent of the group, clearance for all investigated ${ }^{13} \mathrm{C}$-FFA occurred within the first $10 \mathrm{~min}$, with a maximum between 10 and $20 \mathrm{~min}$ and an equilibrated ${ }^{13} \mathrm{C}$ FFA-clearance after 30-90 min of perfusion. A significant increase in ${ }^{13} \mathrm{C}$-FFA-clearance across the placental barrier was observed in the obese group (Fig. 2A-D). Within both the lean and obese groups, clearance followed the order DHA $>$ PA $=$ LA $>$ OA $(0.082,0.053,0.051,0.034 \mathrm{~mL} / \mathrm{min}$ and $0.16,0.087,0.086,0.057 \mathrm{~mL} / \mathrm{min})$ after $20 \mathrm{~min}$, respectively (Fig. 2A-D).

We determined the placental release of endogenous FFA, which were present in placental tissue before the experiment, and the concentration of ${ }^{13} \mathrm{C}$-FFA in fetal perfusates after $90 \mathrm{~min}$. For these calculations we had to exclude two experiments in the lean and one experiment in the obese group due to 3 -fold higher ${ }^{13} \mathrm{C}$-DHA concentration in maternal perfusates at the beginning of the experiment. The placental release of endogenous PA, OA, and LA tend to be lower in placentae of obese compared to lean mothers, notably the endogenous DHA release was significantly lower in the obese group $(p=0.04$, Table 2$)$. The concentration of all applied ${ }^{13} \mathrm{C}$-FFA was elevated in fetal perfusates of obese compared to lean placentae. Interestingly, only ${ }^{13} \mathrm{C}$-PA levels were significantly elevated in obesity.

Next, we calculated the isotopic enrichment of ${ }^{13} \mathrm{C}$ labeled FFA over time in order to state the mobilization kinetic for placental endogenous FFA. The isotopic enrichment in the fetal venous perfusates of ${ }^{13} \mathrm{C}$-labeled FFA was constant over time but varied between the examined FFA. We found the highest enrichment for ${ }^{13} \mathrm{C}$ OA (40-55\%) followed by ${ }^{13} \mathrm{C}-\mathrm{PA},{ }^{13} \mathrm{C}-\mathrm{LA}(20-35 \%)$ and lowest enrichment for ${ }^{13} \mathrm{C}$-DHA with $3-5 \%$ (Fig. 3). Furthermore, the isotopic enrichment for all ${ }^{13} \mathrm{C}$-FFA was higher in obese compared with lean placentae.

No uptake differences of offered ${ }^{13} \mathrm{C}$-FFA into the placental metabolic pool between lean and obese women could be observed (Supplementary Fig. 1).

\section{Discussion}

During intrauterine life a proper development of the fetus depends on maternally derived polyunsaturated FFA. Prior studies have noted a direct link between maternal intake of LC-PUFA with growth measures at birth and development of the fetal brain [30,31]. The placenta coordinates the preferential uptake and transfer of LC-PUFA from the maternal to the fetal circulation, but how the tissue balances this trafficking is still poorly understood. Moreover, most of the studies in this field ignore the capability of the placenta itself to store, metabolize, and to release FAs for different needs. Therefore, an initial objective of this study was to identify how normal placentae compared to placentae from prepregnant obese women differ in respect to FA handling.

The present study is the first to address holistically materno-to-fetal FFA transfer, including to study endogenously provided FFA of the human placenta. We approached this by using transfer data of ${ }^{13} \mathrm{C}$-labeled FFA and applied the results to the recently published mathematical model [22]. In addition, the model approach was extended by DHA, which represents as one of the most important unsaturated FA for fetal development. The strength of the used experimental approach is that it enables simultaneous analysis of transferred labeled FFA and released (endogenous) FFA from the tissue to the fetal circulation. In general, placentae from obese subjects showed a higher clearance for all applied FFA compared to lean in the initial phase of tissue perfusion. More specifically, clearance of DHA was two times higher in obese 
Fig. 2 Clearance of ${ }^{13} \mathrm{C}$-labeled FFA in placentae of lean versus obese women. Direct transfer of ${ }^{13} \mathrm{C}$-labeled A palmitic acid (16:0), $\mathbf{B}$ oleic acid (18:1), C linoleic acid (18:2n6), and $\mathbf{D}$ docosahexaenoic acid $(22: 6 \mathrm{n} 3)$ was determined in lean $(n=7)$ and obese $(n=6)$ placentae by ex vivo perfusion. Kinetic of FFA transfer was investigated utilizing a closed (recirculated) maternal and open (nonrecirculating) fetal circulation. Mean $\left( \pm\right.$ SD) ${ }^{13} \mathrm{C}$ FFA clearance was calculated at time points $0,10,20,30,60$, and $90 \mathrm{~min}$. Two-way ANOVA and Bonferroni post hoc test were performed, one experiment in each group was excluded from the analysis due to missing FFA measurements at one single time point, $* p<0.05$, ** $p<0.01$, and $* * * p<0.001$.

Table 2 Release of endogenous and ${ }^{13} \mathrm{C}$-labeled FFA to the fetal circulation.
A

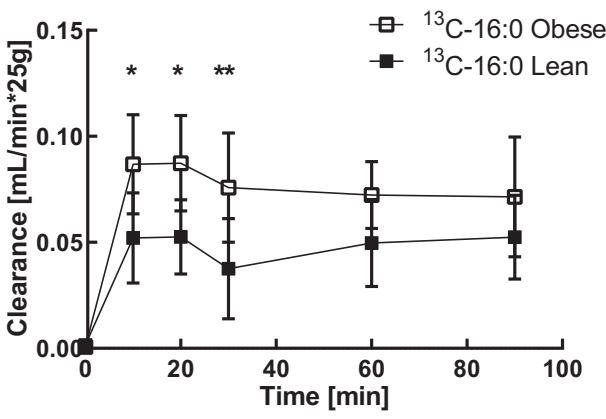

C

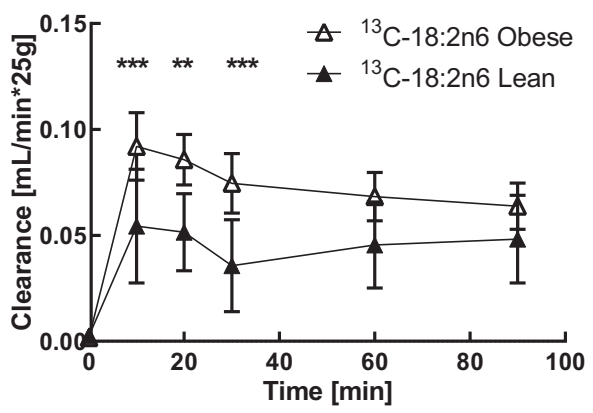

B

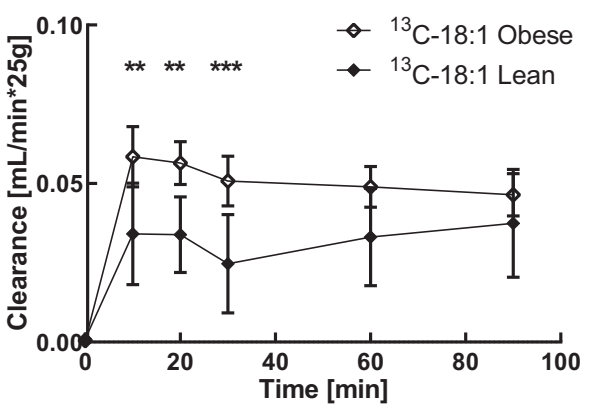

D

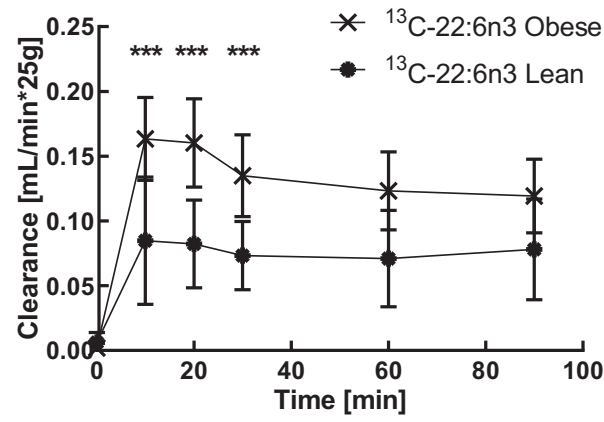

\begin{tabular}{|c|c|c|c|c|c|c|c|c|}
\hline & $16: 0$ & & $18: \ln 9$ & & $18: 2 \mathrm{n} 6$ & & $22: 6 n 3$ & \\
\hline Endoge & us FFA & & & & & & & \\
\hline Lean & 0.642 & $( \pm 0.222)$ & 0.263 & $( \pm 0.098)$ & 0.378 & $( \pm 0.179)$ & 0.072 & $( \pm 0.030)$ \\
\hline Obese & 0.627 & $( \pm 0.295)$ & 0.203 & $( \pm 0.037)$ & 0.3 & $( \pm 0.066)$ & $0.045^{*}$ & $( \pm 0.013)$ \\
\hline${ }^{13} \mathrm{C}-\mathrm{FF}$ & & & & & & & & \\
\hline Lean & 0.118 & $( \pm 0.051)$ & 0.157 & $( \pm 0.056)$ & 0.085 & $( \pm 0.028)$ & 0.001 & $( \pm 0.001)$ \\
\hline Obese & $0.195 *$ & $( \pm 0.055)$ & 0.187 & $( \pm 0.057)$ & 0.108 & $( \pm 0.033)$ & 0.002 & $( \pm 0.001)$ \\
\hline
\end{tabular}

Endogenous FFA and ${ }^{13} \mathrm{C}$-labeled FFA were determined in fetal perfusates after $90 \mathrm{~min}$. Results are adjusted to average perfused cotyledon mass $(25 \mathrm{~g})$ and are expressed as mean $( \pm \mathrm{SD}) \mu \mathrm{mol} / 90 \mathrm{~min}$ of perfused lean $(n=6)$ and obese $(n=6)$ placentae. Differences in endogenous FFA and ${ }^{13} \mathrm{C}$-FFA were tested between lean and obese placentae (Mann-Whitney $U$ test, $* p<0.05$ ). placentae than in lean, suggesting a preferred role of DHA for these specific conditions. However, in general, clearance of all applied FA is low compared to the contribution of tissue released FA. Independently, the general very low clearance of all applied FA emphasizes the importance of lipid accumulation and an altered metabolism in the tissue. In line, it has been shown that maternal BMI is linked to an altered expression of lipid transporters and fatty acid composition in the tissue [32]. The existence of placental intracellular lipid stores has already been shown by us and others $[16,17,33]$. In particular, isolated placental trophoblasts accumulate lipids actively after incubating with FA, a mechanism which is enhanced under pro-inflammatory conditions. Whether an altered cellular FA uptake or metabolic changes may account for these differences cannot be ultimately resolved by using static cell culture model system [34]. One unanticipated finding was that under physiological flow conditions, nonlabeled FFA appear in the fetal circulation in the same time. It is very likely that placental tissue mobilizes endogenous FFA from intracellular lipid stores in order to cover fetal demand under certain metabolic conditions. Release of non-labeled FFA to the fetal compartment exceed $2-3$ times of ${ }^{13} \mathrm{C}$ transferred FA, except for DHA. In the fetal circulation mobilized and released DHA from the placenta exceeds many times over transferred ${ }^{13} \mathrm{C}$-DHA, which was maternally offered in a physiological range (Table 2).

In this study we demonstrate that direct transfer of saturated and unsaturated FFA from the mother to the fetus is elevated in obese compared to lean women. However, maximum membrane flux parameters at the MVM and the $\mathrm{BM}$ obtained from in silico evaluation were similar between lean and obese placentae, suggesting that other variables 
Fig. 3 Isotopic enrichment in fetal effluents. ${ }^{13} \mathrm{C}$-labeled and endogenous FFA were determined in fetal venous samples A palmitic acid (16:0), B oleic acid (18:1), C linoleic acid (18:2n6), and $\mathbf{D}$ docosahexaenoic acid (22:6n3). Mean $( \pm \mathrm{SD}){ }^{13} \mathrm{C}$-isotopic enrichment in perfusion experiments, lean $(n=8)$ and obese $(n=7)$ placentae were calculated at time points 0,10 , $20,30,60$, and $90 \mathrm{~min}$ and is expressed as percentage.
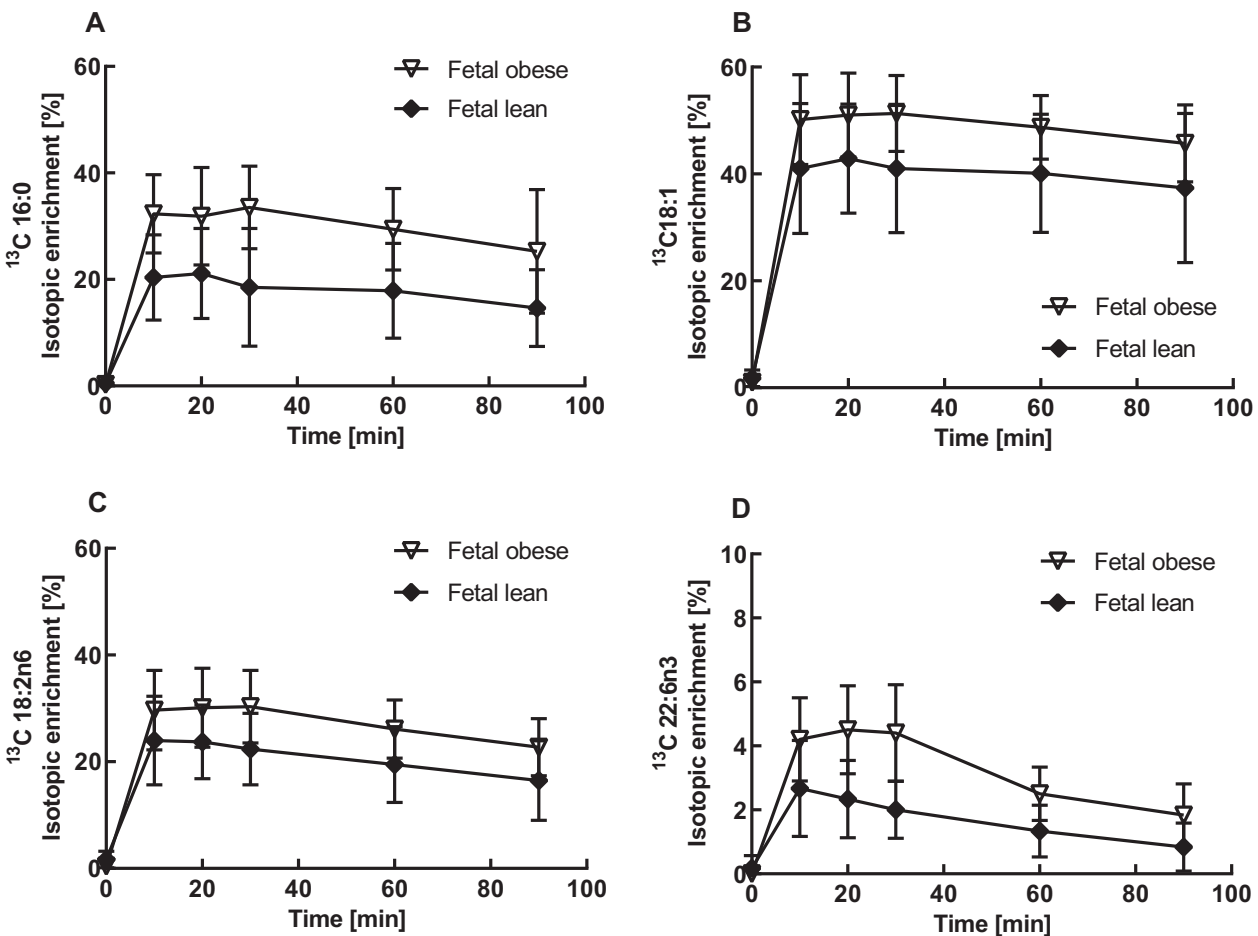

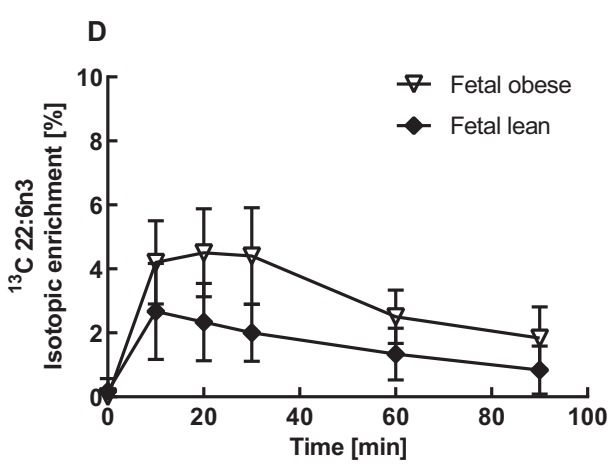

than membrane fluxes may account for this effect. Interestingly, the rate constant for the metabolic DHAaccumulation in the model $\left(\mathrm{k}_{\mathrm{acc}}\right)$ was lower in obese compared to lean placentae, indicating a favorable direct transfer of DHA to the fetal circulation under this specific metabolic condition, which matches our experimental data.

Recently, Gazquez et al. published two in vivo tracer studies where lean and obese women received ${ }^{13} \mathrm{C}$-FFA orally prior to cesarean section $[35,36]$. Although these and our studies touching a similar area, a direct comparison of absolute FA-levels or ratio of ${ }^{13} \mathrm{C}-\mathrm{FA}$ enrichment in the placenta is inaccurate, because of different timing for collected samples. First, labeled FFA were administered orally $12 \mathrm{~h}$ before delivery. Second, while cord blood was taken directly after birth, last maternal blood sample was taken $2 \mathrm{~h}$ before delivery which cannot be defined as matched samples. In contrast, our experimental setting enabled to collect maternal and fetal samples simultaneously at distinct time points after administrating ${ }^{13} \mathrm{C}-\mathrm{FA}$. Interestingly, in both studies compared to lean placentae, the trend for lower DHA levels in placenta and cord blood affected by obesity is identifiable. In our study decreased levels of endogenous DHA were seen in obesity, and weighs heavier than the transferred ${ }^{13} \mathrm{C}$-DHA (Table 2).

The other examined FA, ${ }^{13} \mathrm{C}$-stearic acid, ${ }^{13} \mathrm{C}$-palmitic acid, and ${ }^{13} \mathrm{C}$-oleic acid, showed a materno-fetal transfer of around $\sim 1 \%$ [35]. These values are comparable to our results obtained for palmitic acid by ex vivo perfusion approach. However, the transfer kinetics for oleic acid were slightly lower and that of DHA, which was significantly higher by our ex vivo approach. That was previously also observed by Haggarty et al. [37], and raised a concept of FA-transfer across the placenta that involves fatty acid transport proteins (FATP) and fatty acid binding proteins (FABP) [21].

Our approach using the ex vivo placental perfusion technique in combination with in silico modeling highlights the importance of including the endogenous capability of the placenta to mobilize and release (unsaturated) FA, beside materno-to-fetal transfer, which overall cannot be fully accessed by in vivo studies. In our in silico model ${ }^{13} \mathrm{C}$ FFA transfer to the fetal reservoir and accumulation in the placental lipid pool was addressed, but there was no relevant ${ }^{13} \mathrm{C}$-FFA release from the placental lipid pool. Since the uptake parameter exceeds the release parameter by over two orders of magnitude, remobilization of ${ }^{13} \mathrm{C}$-FFA may take longer than $90 \mathrm{~min}$. A limitation of the in silico model is that it could not cover mobilization and release of endogenous FFA present in placental tissue before the experiment, as we observed it in the ex vivo experiments.

The low number of investigated subjects is certainly a methodological limitation of our study. Nevertheless, in comparison to other ex vivo perfusion studies, enrolled tissue perfusions are of high experimental quality [25] and the number of investigated placentas are comparative [38]. Unfortunately, we could not assess metabolic and inflammatory parameters of obese women. However, as we know from other studies, surrogate clinical parameters like weight, height of the offspring, or placental morphology might not reflect its metabolic condition [39, 40]. It is possible that the increased 


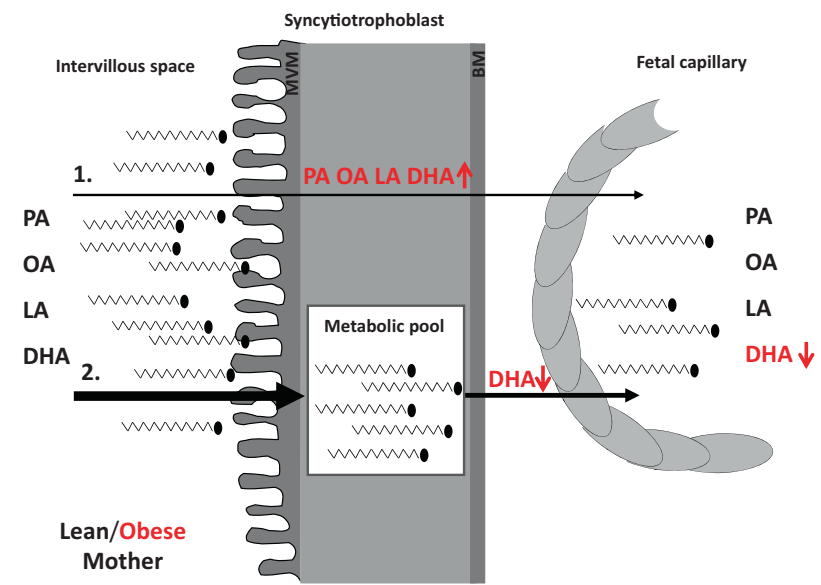

Fig. 4 Model for FFA transfer across the placental barrier. (1) FFA derived from maternal plasma is transferred directly across the placental syncytiotrophoblast to the placental-fetal endothelium. In placentae of obese women transfer rates for all examined FFA are elevated (red arrow). (2) Simultaneously, maternal FFA are taken up by the placenta and mass balance calculations suggest an enrichment of FFA within the tissue (metabolic pool). Out of the metabolic pool, FFA may be released to the fetal circulation. In fact, mobilization of DHA was reduced in placentae of obese women, resulting in lower DHA levels in the fetal capillaries/circulation. MVM microvillous membrane, BM basal membrane, PA palmitic acid, DHA docosahexaenoic acid, LA linoleic acid, and OA oleic acid.

FFA flux across obese placentae contributes to the elevated lipid accumulation in fetal adipose tissue and therefore altering body composition rather than birth weight [4].

To conclude, the importance of this area is illustrated by our findings in which only a small proportion of FFA are directly transferred across the placenta in comparison to the FFA proportion released by the metabolic pool in the same time. The exogenous FFA transfer capacity in the syncytium reaches a maximum in a short time period implying that this process contributes only slightly to the materno-tofetal FA supply. In contrast, tissue stored FA (mainly in phospholipids) are an efficiently and quickly available source of FA for the release to the fetus. Overall, our data suggest an expanded compartment mediated concept of FA uptake, storage, and delivery to the fetus in the human placenta (Fig. 4). Specific placental metabolic alterations seem to contribute more to the unfavorable newborn outcome than thought before, and further prime the offspring towards the generation spanning obesity cycle [41]. Breaking the cycle of intergenerational obesity needs more prospectively planned cohort studies. Together, our applied combined in silico and ex vivo approach presented in this study provides a comprehensive integrated approach to study materno-to-fetal lipid trafficking in normal but, more importantly, in pathophysiological pregnancies.

Acknowledgements GC-MS analysis was performed with the help and guidance of Harald Köfeler, $\mathrm{PhD}$ and Stefanie Rappold from the Core
Facility for Mass Spectrometry, Center for Medical Research, Medical University of Graz, Austria. We want to thank Bettina Amtmann and Petra Winkler for communication between research laboratory and delivery room and Susanne Kopp for technical assistance. This work was funded by European Union's Seventh Framework Program (FP7/ 2007-2013), project Early Nutrition under grant agreement $n^{\circ} 289346$. Birgit Hirschmugl received funding from the Anniversary Fund of the Oesterreichiche Nationalbank (grant no. 18181) and the Medical University of Graz by the $\mathrm{PhD}$ Program Molecular Fundamentals of Inflammation (DK-MOLIN, funded by the Austrian Science Fund FWF (W1241)). This work was further supported by Soroptimist International, Club Goldes Südsteiermark. Simone Perazzolo received funding from the Institute of Life Sciences, Southampton.

Author contributions RML, BGS, GD, and CW conceived and designed the study. BH performed the experiments. BH and MG validated perfusion quality control parameters. $\mathrm{BH}, \mathrm{SP}$, and BGS analyzed data and performed statistics. RML, BGS, SP, BH, and CW interpreted data sets. $\mathrm{BH}, \mathrm{SP}$, and $\mathrm{CW}$ wrote the manuscript. RML, BGS, and GD revised the paper. All authors read and approved the manuscript.

\section{Compliance with ethical standards}

Conflict of interest The authors declare no competing interests.

Publisher's note Springer Nature remains neutral with regard to jurisdictional claims in published maps and institutional affiliations.

Open Access This article is licensed under a Creative Commons Attribution 4.0 International License, which permits use, sharing, adaptation, distribution and reproduction in any medium or format, as long as you give appropriate credit to the original author(s) and the source, provide a link to the Creative Commons license, and indicate if changes were made. The images or other third party material in this article are included in the article's Creative Commons license, unless indicated otherwise in a credit line to the material. If material is not included in the article's Creative Commons license and your intended use is not permitted by statutory regulation or exceeds the permitted use, you will need to obtain permission directly from the copyright holder. To view a copy of this license, visit http://creativecommons. org/licenses/by/4.0/.

\section{References}

1. World Health Organization. Obesity and Overweight - Fact sheets. 2016; 2016.

2. Metsala J, Stach-Lempinen B, Gissler M, Eriksson JG, Koivusalo S. Risk of pregnancy complications in relation to maternal prepregnancy body mass index: population-based study from Finland 2006-10. Paediatr Perinat Epidemiol. 2016;30:28-37.

3. Carlson NS, Hernandez TL, Hurt KJ. Parturition dysfunction in obesity: time to target the pathobiology. Reprod Biol Endocrinol. 2015; $13: 135$

4. Sewell MF, Huston-Presley L, Super DM, Catalano P. Increased neonatal fat mass, not lean body mass, is associated with maternal obesity. Am J Obstet Gynecol. 2006;195:1100-3.

5. Ehrenberg HM, Mercer BM, Catalano PM. The influence of obesity and diabetes on the prevalence of macrosomia. Am J Obstet Gynecol. 2004;191:964-8.

6. Gaillard R, Durmuş B, Hofman A, Mackenbach JP, Steegers EA, Jaddoe VW. Risk factors and outcomes of maternal obesity and excessive weight gain during pregnancy. Obesity (Silver Spring). 2013;21:1046-55. 
7. Whitaker RC. Predicting preschooler obesity at birth: the role of maternal obesity in early pregnancy. Pediatrics. 2004;114:e29-36.

8. Eriksson J, Forsen T, Tuomilehto J, Osmond C, Barker D. Size at birth, childhood growth and obesity in adult life. Int J Obes Relat Metab Disord. 2001;25:735-40.

9. Dube E, Gravel A, Martin C, Desparois G, Moussa I, EthierChiasson M, et al. Modulation of fatty acid transport and metabolism by maternal obesity in the human full-term placenta. Biol Reprod. 2012;87:1-11.

10. Ramsay JE, Ferrell WR, Crawford L, Wallace AM, Greer IA, Sattar N. Maternal obesity is associated with dysregulation of metabolic, vascular, and inflammatory pathways. The $\mathrm{J}$ of Clin Endocrinol \& Metab. 2002;87:4231-7.

11. Catalano PM, Presley L, Minium J, Hauguel-de Mouzon S. Fetuses of obese mothers develop insulin resistance in utero. Diabetes Care. 2009;32:1076-80.

12. Aye IL, Lager S, Ramirez VI, Gaccioli F, Dudley DJ, Jansson T, et al. Increasing maternal body mass index is associated with systemic inflammation in the mother and the activation of distinct placental inflammatory pathways. Biol Reprod. 2014;90:129.

13. Tinius RA, Cahill AG, Strand EA, Cade WT. Altered maternal lipid metabolism is associated with higher inflammation in obese women during late pregnancy. Integr Obes Diabetes. 2015;2:168-75.

14. Innis SM. Dietary (n-3) fatty acids and brain development. J Nutr. 2007;137:855-9.

15. Saben J, Lindsey F, Zhong Y, Thakali K, Badger TM, Andres A, et al. Maternal obesity is associated with a lipotoxic placental environment. Placenta. 2014;35:171-7.

16. Calabuig-Navarro V, Haghiac M, Minium J, Glazebrook $P$, Ranasinghe GC, Hoppel C, et al. Effect of maternal obesity on placental lipid metabolism. Endocrinology. 2017;158:2543-55.

17. Hirschmugl B, Desoye G, Catalano P, Klymiuk I, Scharnagl H, Payr $\mathrm{S}$, et al. Maternal obesity modulates intracellular lipid turnover in the human term placenta. Int J Obes (Lond). 2017;41:317-23.

18. Mele J, Muralimanoharan S, Maloyan A, Myatt L. Impaired mitochondrial function in human placenta with increased maternal adiposity. Am J Physiol Endocrinol Metab. 2014;307:E419-25.

19. Lager S, Ramirez VI, Gaccioli F, Jang B, Jansson T, Powell TL. Protein expression of fatty acid transporter 2 is polarized to the trophoblast basal plasma membrane and increased in placentas from overweight/obese women. Placenta. 2016;40:60-66.

20. Diaz P, Harris J, Rosario FJ, Powell TL, Jansson T. Increased placental fatty acid transporter 6 and binding protein 3 expression and fetal liver lipid accumulation in a mouse model of obesity in pregnancy. Am J Physiol Regul Integr Comp Physiol. 2015;309: R1569-77.

21. Herrera E, Desoye G. Maternal and fetal lipid metabolism under normal and gestational diabetic conditions. Horm Mol Biol Clin Investig. 2016;26:109-27.

22. Perazzolo S, Hirschmugl B, Wadsack C, Desoye G, Lewis RM, Sengers BG. The influence of placental metabolism on fatty acid transfer to the fetus. J Lipid Res. 2017;58:443-54.

23. Haggarty P, Ashton J, Joynson M, Abramovich DR, Page K. Effect of maternal polyunsaturated fatty acid concentration on transport by the human placenta. Biol Neonate. 1999;75:350-9.

24. Schneider H, Huch A. Dual in vitro perfusion of an isolated lobe of human placenta: method and instrumenatation. In: Schneider H, Dancis J, editors. In vitro perfusion of human placental tissue. Basel, Switzerland: Karger; 1985. p.40-7. .
25. Mathiesen L, Mose T, Morck TJ, Nielsen JK, Nielsen LK, Maroun LL, et al. Quality assessment of a placental perfusion protocol. Reprod Toxicol. 2010;30:138-46.

26. Challier JC, D'Athis P, Guerre-Millo M, Nandakumaran M. Flowdependent transfer of antipyrine in the human placenta in vitro. Reprod Nutr Dev. 1983;23:41-50.

27. Annola K, Heikkinen AT, Partanen H, Woodhouse H, Segerback $\mathrm{D}$, Vahakangas K. Transplacental transfer of nitrosodimethylamine in perfused human placenta. Placenta. 2009;30:277-83.

28. Matyash V, Liebisch G, Kurzchalia TV, Shevchenko A, Schwudke D. Lipid extraction by methyl-tert-butyl ether for highthroughput lipidomics. J Lipid Res. 2008;49:1137-46.

29. Fuchs CD, Claudel T, Kumari P, Haemmerle G, Pollheimer MJ, Stojakovic T, et al. Absence of adipose triglyceride lipase protects from hepatic endoplasmic reticulum stress in mice. Hepatology. 2012;56:270-80.

30. Szajewska H, Horvath A, Koletzko B. Effect of n-3 long-chain polyunsaturated fatty acid supplementation of women with lowrisk pregnancies on pregnancy outcomes and growth measures at birth: a meta-analysis of randomized controlled trials. Am J Clin Nutr. 2006;83:1337-44.

31. Helland IB, Smith L, Saarem K, Saugstad OD, Drevon CA. Maternal supplementation with very-long-chain n-3 fatty acids during pregnancy and lactation augments children's IQ at 4 years of age. Pediatrics. 2003;111:e39-44.

32. Segura MT, Demmelmair H, Krauss-Etschmann S, Nathan P, Dehmel S, Padilla MC, et al. Maternal BMI and gestational diabetes alter placental lipid transporters and fatty acid composition. Placenta. 2017;57:144-51.

33. Watkins OC, Islam MO, Selvam P, Pillai RA, Cazenave-Gassiot A, Bendt AK, et al. Metabolism of 13C-labeled fatty acids in term human placental explants by liquid chromatography-mass spectrometry. Endocrinology. 2019;160:1394-408.

34. Lager S, Jansson N, Olsson AL, Wennergren M, Jansson T, Powell TL. Effect of IL-6 and TNF-alpha on fatty acid uptake in cultured human primary trophoblast cells. Placenta. 2011;32:121-7.

35. Gazquez A, Prieto-Sanchez MT, Blanco-Carnero JE, van Harskamp D, Perazzolo S, Oosterink JE, et al. In vivo kinetic study of materno-fetal fatty acid transfer in obese and normal weight pregnant women. J Physiol. 2019;597:4959-73.

36. Gazquez A, Prieto-Sanchez MT, Blanco-Carnero JE, RuizPalacios M, Nieto A, van Harskamp D, et al. Altered maternofetal transfer of 13C-polyunsaturated fatty acids in obese pregnant women. Clin Nutr. 2020;39:1101-7.

37. Haggarty P, Page K, Abramovich DR, Ashton J, Brown D. Longchain polyunsaturated fatty acid transport across the perfused human placenta. Placenta. 1997;18:635-42.

38. Kuhn DC, Crawford M. Placental essential fatty acid transport and prostaglandin synthesis. Prog Lipid Res. 1986;25:345-53.

39. Farah N, Stuart B, Donnelly V, Kennelly MM, Turner MJ. The influence of maternal body composition on birth weight. Eur $\mathbf{J}$ Obstet Gynecol Reprod Biol. 2011;157:14-17.

40. Mitanchez D, Jacqueminet S, Nizard J, Tanguy ML, Ciangura C, Lacorte JM, et al. Effect of maternal obesity on birthweight and neonatal fat mass: a prospective clinical trial. PLoS ONE. 2017; 12:e181307.

41. Catalano PM. Obesity and pregnancy-the propagation of a viscous cycle? J Clin Endocrinol Metab. 2003;88:3505-6. 